\title{
An experimental model of evaporative cooling system with forced circulation of coolant for high-performance electronic components
}

\author{
Konstantin Stepanov ${ }^{1}$, Dmitry Mukhin ${ }^{1}$, Karapet Eloyan ${ }^{1}$, Egor Tkachenko ${ }^{1,2}$ \\ ${ }^{1}$ Institute of Thermophysics SB RAS, Novosibirsk, Russia \\ ${ }^{2}$ Novosibirsk State University, Novosibirsk, Russia
}

\begin{abstract}
A promising way to remove heat fluxes from the surfaces of electronic devices with high heat generation is the use of evaporating thin liquid film, moving under the action of gas flow in a channel. On the basis of the studies conducted previously, we have developed a pilot model of the experimentalindustrial prototype for removing heat fluxes with densities of up to $1000 \mathrm{~W} / \mathrm{cm}^{2}$ from the surface of the heat-stressed element with dimensions $10 \times 10 \mathrm{~mm}^{2}$. Testing of the model has proved its efficiency in continuous operation.
\end{abstract}

\section{Introduction}

Currently, the global industry is ready to produce high-performance electronic components where the heat flux density at individual sites can reach $1000 \mathrm{~W} / \mathrm{cm}^{2}$ and higher [1]. However, the introduction and use of these devices faces challenges of removing such high specific heat fluxes into the ambient medium. One of the promising ways of removing large heat fluxes from the surface of heat-stressed elements of electronic devices is the use of evaporating thin layer of liquid film, moving under the action of the gas flow in a flat channel $[2,3,4]$.

Recent experimental investigations conducted in works $[5,6]$ proved possible removal of heat fluxes with density of up to $870 \mathrm{~W} / \mathrm{cm}^{2}$ from the heating area of $1 \times 1 \mathrm{~cm}^{2}$ using this method. The works $[7,8]$ showed that such a system can operate stably in a wide range of the channel heights $(0.17-2.00 \mathrm{~mm})$ and angles of the channel inclination to the horizon $\left(0-360^{\circ}\right)$. In [9] a 3D non-stationary mathematical model of joint motion of evaporating liquid film and co-current gas flow in a microchannel with local heating has been developed, taking into account a deformable gas-liquid interface, convective heat transfer in the liquid and gas phases as well as temperature dependence of surface tension and liquid viscosity. The performed investigations have resulted in the development of a promising model of evaporative cooling system for electronic devices with high heat flux removal and forced circulation of coolants. The model is an experimental-industrial prototype of cooling system with closed loops of coolants. 


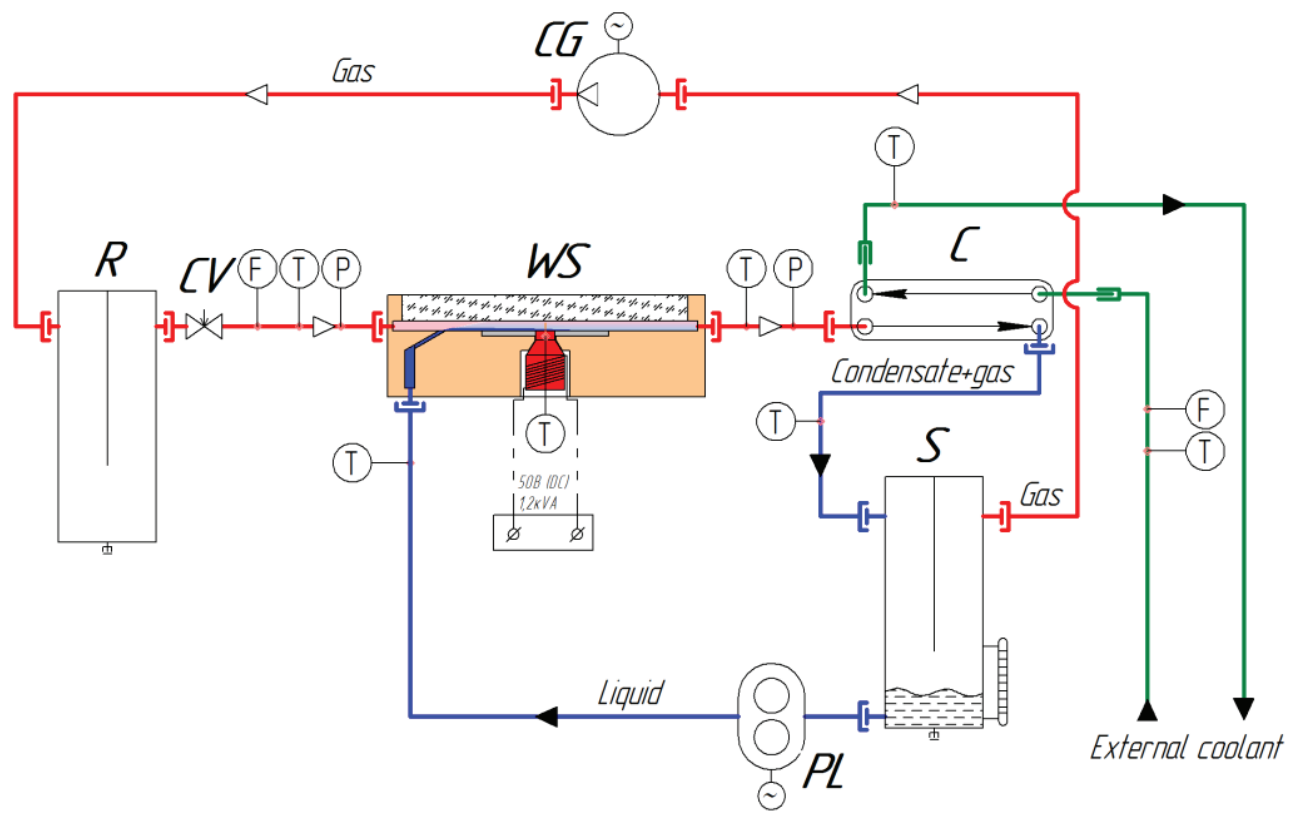

Fig. 1. Schematic diagram of the experimental model of evaporative cooling system: WS - working section; C - condenser; $\mathrm{S}$ - separator; $\mathrm{R}$ - receiver; CV - control valve; $\mathrm{PL}$ - liquid pump; CG - gas vacuum pump-compressor.

\section{Experimental model}

A schematic diagram of the experimental model of evaporative cooling system is shown in Fig. 1. The principle of heat removal from the surface simulating the heated wall of heatstressed electronic component is based on the use of intensively evaporating thin liquid film, moving under the action of cocurrent gas flow or vapor-gas mixture in a slot mini- or microchannel. This method of cooling allows acheaving of substantially increased critical heat fluxes needed for a liquid film to rupture and also removing high heat fluxes from surfaces with small area.

To ensure continuous operation of the experimental model gas and liquid circulate in closed loops. In the loops different pressures can be maintained: atmospheric, vacuum or excess pressure. In the gas loop the gas is purified from impurity - air or nitrogen with a small amount of liquid vapor. To enable gas circulation in the loop, vacuum pumpcompressor CG is used. In the fluid circuit the purified liquid is used, i.e. water, ethanol or HFE-7100. Fluid is circulated using high-precision gear pump PL. After entering into the working section WS the liquid moves under the action of the cocurrent gas flow.

In the working section, gas and liquid move in a slot channel with the height of $1.0 \mathrm{~mm}$ and the width of $11 \mathrm{~mm}$. The slot channel is formed between the working surface and a viewing glass, and the channel height is set by a spacer plate (the channel height can be changed from 0.1 to $2.0 \mathrm{~mm}$ ). The fluid flows through the channel in the form of a film, entrained by the gas flow. To form a liquid film with certain thickness in the working section a slit nozzle is used; it is formed by a wedge-shaped knife and the working plate, over which the film is distributed (see Fig. 2). 


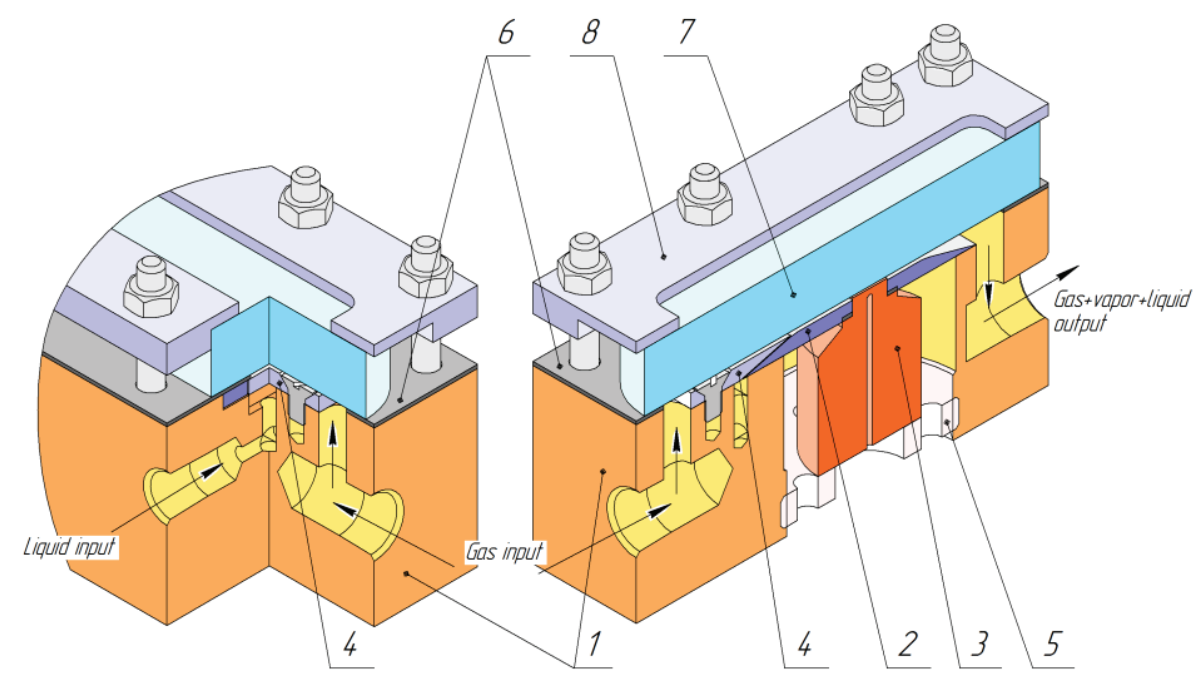

Fig. 2. Design of the working section WS: 1 - housing; 2 - working plate; 3 - the local heater (copper rod); 4 - a wedge-shaped knife; 5 - holder; 6 - spacer plate; 7 - viewing glass; 8 - flange.

The formed liquid film is supplied to the center of the slot channel, to the zone of the heat-stressed electronic component simulator - the local heater, where it is evaporating, due to heat flux supply to the local heater. The resulting vapor is carried away by the gas passing over the liquid film. At the end of the slot channel the vapor-gas mixture together with the liquid is supplied through the loop to the condenser C (Fig. 1), where vapor condensation of the evaporated liquid occurs. After the condenser $\mathrm{C}$ the gas-liquid mixture goes to a separator $\mathrm{S}$, where the separation of liquid and gas takes place. The gas then enters the suction line of the pump-compressor CG, and the liquid accumulates in the lower part of the separator and enters the pump PL.

The local heater is a copper rod, heated by a nichrome wire (not shown in Fig. 2), by means of passed electric current. The wire is helically wound around the core of the local heater. The dimensions of the flat surface of the local heater are $10 \times 10 \mathrm{~mm}^{2}$. Power is supplied to the heating element using a direct current source. The heat flux on the local heater is adjusted by changing the current strength.

The removal of condensation heat from the condenser $\mathrm{C}$ is realized by external coolant - water (or other liquid). The flow of circulating gas is regulated by the frequency converter of pump-compressor $\mathrm{CG}$, and the control valve $\mathrm{CV}$, mounted at the inlet of the working section. The flow rate of the circulating fluid is regulated by means of the control unit of the liquid pump PL.

Measurements of temperatures, pressures and flow rates are performed in a centralized measurement system, consisting of measurement modules and a personal computer.

\section{Test results}

The pilot model of the evaporative cooling system was used to test the removal of the heat flux with density of up to $1000 \mathrm{~W} / \mathrm{cm}^{2}$ from the local heater of the working section. The used working fluid was distilled water, and the used working gas was purified air. The used external coolant was tap water. The parameters of the pilot model are given in Table 1 . 
Table 1. The results of the test of experimental model.

\begin{tabular}{|l|c|}
\hline \multicolumn{1}{|c|}{ Parameter } & Value \\
\hline Density of the heat flux, W/cm ${ }^{2}$ & $0 \div 1000$ \\
\hline Volumetric flow rate of circulating fluid, ml/min & $100 \div 120$ \\
\hline Volumetric flow rate of circulating gas, 1/min & $45 \div 50$ \\
\hline Temperature of liquid at the inlet the working section, ${ }^{\circ} \mathrm{C}$ & $28 \div 30$ \\
\hline Gas temperature at the inlet of the working section, ${ }^{\circ} \mathrm{C}$ & $40 \div 45$ \\
\hline Temperature of the gas mixture at the output from the working section, ${ }^{\circ} \mathrm{C}$ & $55 \div 60$ \\
\hline Absolute gas pressure at the inlet of the working area, Bar & $1.3 \div 1.4$ \\
Absolute pressure of the gas mixture at the output of the working section, Bar & $1.0 \div 1.1$ \\
\hline Temperature of the external coolant at the inlet of the condenser, ${ }^{\circ} \mathrm{C}$ & $23 \div 25$ \\
\hline
\end{tabular}

Tests have confirmed the efficiency of the experimental model in a continuous operation at the maximum heat flux for 50 minutes. The local surface temperature of the heater in the zone of evaporative cooling did not exceed $140{ }^{\circ} \mathrm{C}$. Visual observations of the local heater surface have revealed no formation of stationary dry spots in the range of heat fluxes up to $1000 \mathrm{~W} / \mathrm{cm}^{2}$.

\section{Conclusions}

Initial tests of the experimental model of evaporative cooling system with forced circulation of coolants for electronic devices with high heat emission have confirmed the possibility of continuous discharge of the heat flux with density of up to $1000 \mathrm{~W} / \mathrm{cm}^{2}$ from a flat surface of heat-stressed element with the size of $1 \times 1 \mathrm{~cm}^{2}$ at relatively low flow rates of liquid and gas.

The experimental setup realized in a closed cycle of coolants has the potential of providing even higher heat fluxes. Further stages of model testing will be realized to develop this potential.

This work was supported by the Ministry of Education and Science of Russia (Agreement No 14.604.21.0053, project identifier RFMEFI60414X0053).

\section{References}

1. A. Bar-Cohen, C. Holloway, Journal of Physics: Conference Series, 745, 022002 (2016)

2. O.A. Kabov and D.V. Zaitsev, Multiphase Science and Technology, 21, 249-266 (2009)

3. D.V. Zaitsev, D.A. Rodionov, O.A. Kabov, Technical Physics Letters, 35, 680-682 (2009)

4. O.A. Kabov, D.V. Zaitsev, V.V. Cheverda, A. Bar-Cohen, Experimental Thermal and Fluid Science, 35, 825 (2011)

5. D. Zaitsev, E. Tkachenko, E. Orlik, O. Kabov, MATEC Web of Conferences, 92, 01037 (2016)

6. E.M. Tkachenko, D.V. Zaitsev, E.V. Orlik, O.A. Kabov, Journal of Physics: Conference Series, 754, 032019 (2016)

7. D. Zaitsev, O. Kabov, MATEC Web of Conferences, 84, 00043 (2016)

8. E.M. Tkachenko, D.V. Zaitsev, MATEC Web of Conferences, 72, 01114 (2016)

9. Y. Kabova, V.V. Kuznetsov, O. Kabov, T. Gambaryan-Roisman, P. Stephan, International Journal of Heat and Mass Transfer, 68, 527-541 (2014) 